\title{
Droplet impact on a thin fluid layer
}

\author{
By S. D. HOWISON ${ }^{1}$, J. R. OCKENDON ${ }^{1}$, J. M. OLIVER I $^{2}$, \\ R. PURVIS ${ }^{3}$ AND F. T. SMITH ${ }^{4}$ \\ ${ }^{1}$ Mathematical Institute, 24-29 St. Giles', Oxford OX1 3LB, UK \\ ${ }^{2}$ School of Mathematical Sciences, University of Nottingham, Nottingham NG7 2RD, UK \\ ${ }^{3}$ School of Mathematics, University of East Anglia, Norwich NR4 7TJ, UK \\ ${ }^{4}$ The Department of Mathematics, University College London, Gower Street, London WC1E 6BT, UK
}

(Received 9 August 2004 and in revised form 20 May 2005)

The initial stages of high-velocity droplet impact on a shallow water layer are described, with special emphasis given to the spray jet mechanics. Four stages of impact are delineated, with appropriate scalings, and the successively more important influence of the base is analysed. In particular, there is a finite time before which part of the water in the layer remains under the droplet and after which all of the layer is ejected in the splash jet.

\section{Introduction}

Based on the ideas of Wagner (1932), there is a well-established theory for the initial stages of the high-velocity impact between rigid bodies and half-spaces of inviscid incompressible liquid; the resulting flow gives rise to a high pressure acting on part of the impactor. One of the interesting predictions is that when the 'deadrise angle' between the tangent to the impactor and the initial boundary of the half-space is small, splash jets emerge from the perimeter of the high-pressure region which, relative to the size of the impactor, are of thickness of the order of the deadrise angle squared. This asymptotic scaling allows so-called 'Wagner conditions' to be applied near the jet roots and these conditions then determine the evolution of the free surfaces away from the jet roots. In Oliver (2002), Howison, Ockendon \& Oliver (2002, 2004) and Howison \& Oliver (2004), the theory has been extended to the case of impact on a confined layer of finite thickness, even to the stage where the layer is a film whose thickness compared to the size of the impactor is of the order of the deadrise angle, which was first studied by Korobkin (1995).

Less theory is available for analogous liquid-liquid impacts. However, the initial impact between equal droplets is a trivial example of Wagner theory and, as explained in Howison, Ockendon \& Wilson (1991), some features of unequal droplet impact can be discerned when Wagner theory is just taken to the lowest order in the deadrise angle. In the presence of a base, the scenario that emerges when the droplet radius, $R$, is much greater than the layer thickness, $H$, and when time $t \ll R / U$, where $U$ is the impact velocity, is that of figure 1 . To lowest order as $t \rightarrow 0^{+}$the splash jets are horizontal and have thicknesses of $O\left(t^{3 / 2}\right)$ near the 'turnover' points, where the free surfaces are vertical. This may be compared with the case of impact on a dry solid base, which is also described by Wagner theory and leads to the flow in figure 2. In both cases the inner regions containing the turnover points move in an outer region of size of $O\left(t^{1 / 2}\right)$ in which the deadrise angle of the undisturbed droplet is of $O\left(t^{1 / 2}\right)$ 
(a)

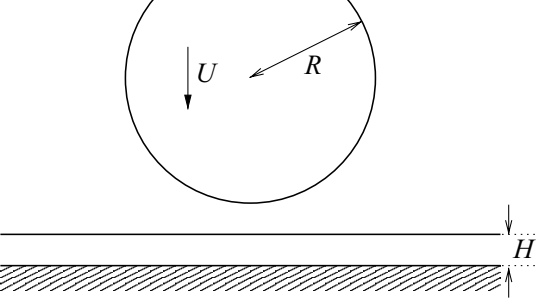

(b)

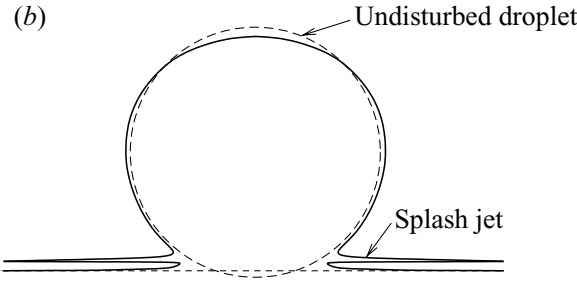



FIGURE 1. Schematic of the normal impact of a two-dimensional cylindrical droplet on a thin fluid layer $(a)$ before impact $t<0$ and $(b)$ just after impact $0<t \ll R / U$.

(a)

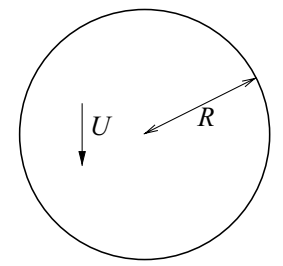

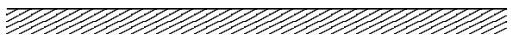

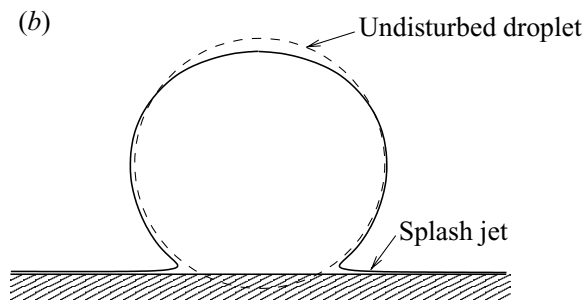

FIGURE 2. Schematic of the normal impact of a two-dimensional cylindrical droplet on a dry base $(a)$ before impact $t<0$ and $(b)$ just after impact $0<t \ll R / U$.

as $t \rightarrow 0^{+}$. Moreover both theories lead to the interesting prediction that the splash jets extend instantaneously to infinity at $t=0^{+}$.

The study of aircraft icing by Gent, Dart \& Cansdale (2000) motivates the development of the theory to encompass the impact between a liquid drop and a thin film on a solid base, with the thickness and velocity of the splash sheet being of especial interest. Hence, in this paper, we present such a theory for the case when the layer thickness is small compared with the droplet radius, thereby unifying the scenarios of figures 1 and 2. Although there is only one important geometric parameter in our theory, in $\S 3$ we show that four distinguished limits emerge as the impact evolves, and these are described sequentially in $\$ 4-\S 6$, with some of the technical details relegated to the Appendices. The theoretical and physical implications of our theory are reviewed in $\S 7$. Although all the discussion in earlier sections concerns two-dimensional flow, we show that the impact of an axisymmetric drop can be analysed in exactly the same way; the only minor difference is that, in the axisymmetric case, the solution of a particular boundary-value sub-problem that arises in the asymptotic analysis cannot be found in a simple closed form. In $\S 7$ we also discuss the practical relevance of the model. In particular, we give a scaling argument from which it is possible to bound the time scales over which viscosity and surface tension (both of which are neglected in our model) have a significant effect. We also make a comparison with the experimental results of Thoroddsen (2002) on the impact of a drop on a liquid half-space, showing that, with his set-up, our model may be expected to be valid for all except extremely short times (at the most, microseconds) from initial impact. 


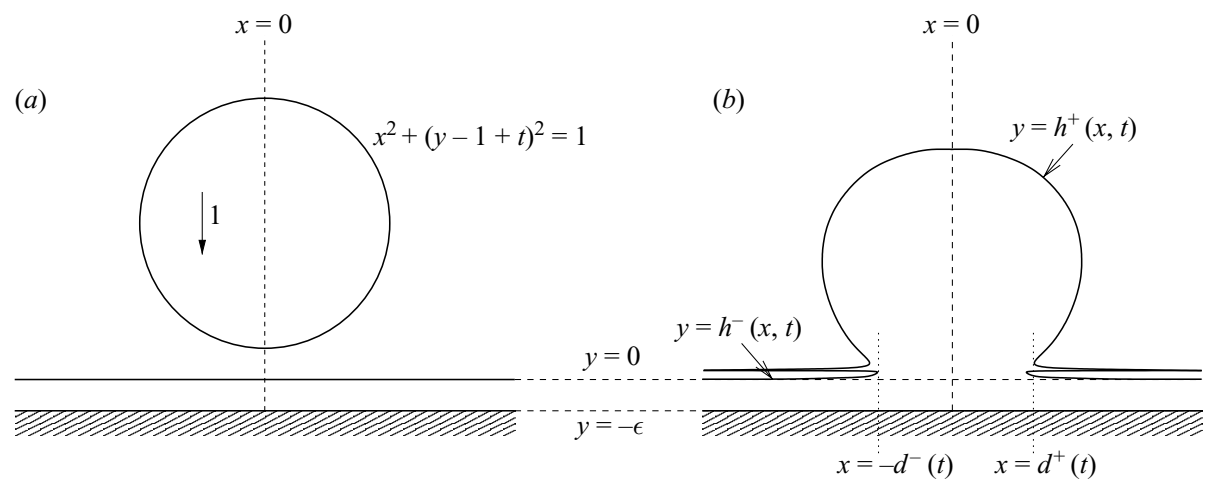

FIGURE 3. Geometry of the dimensionless model problem for $(a) t<0$ and $(b) 0<t \ll 1$. The points $x=-d^{+}(t)$ and $x=d^{-}(t)$ are not labelled.

\section{Problem statement}

We first consider the two-dimensional normal impact depicted in figure 1, where a cylindrical water droplet whose radius is $R$ impacts with speed $U$ on a water layer of thickness $H$. We only consider the case when $\epsilon=H / R$ is small and $U$ is so large that viscosity, gravity and surface tension effects are negligible. We also work on time scales that are long compared to the acoustic time scale in the water and we neglect the effect of the air trapped between the droplet and the layer.

When distances are made dimensionless with $R$ and time with $R / U$, we obtain the configuration in figure 3 , with the nominal penetration depth (i.e. the ordinate of the minimum of the unperturbed droplet boundary) being equal to $-t$. We denote the perturbed droplet surface, including the upper side of the jets, by $y=h^{+}(x, t)$ and the perturbed layer surface, including the lower side of the jets, by $y=h^{-}(x, t)$, both of these being symmetric about $x=0$ and multi-valued. We denote the $x$-coordinates of the four turnover points, where the free surfaces are vertical, by $x= \pm d^{ \pm}(t)$ as indicated in figure $3(b)$ and anticipate that except at the very end of the paper, in all the flows we consider, the turnover points of the lower free surfaces are close (in a sense to be made precise) to those of the upper free surface. In what follows, we use $x= \pm d^{+}(t)$ as reference points for the turnover regions.

Scaling the velocity potential with $U R$, the dimensionless model problem is

$$
\frac{\partial^{2} \phi}{\partial x^{2}}+\frac{\partial^{2} \phi}{\partial y^{2}}=0,
$$

in the fluid region of figure $3(b)$, with

$$
\frac{\partial \phi}{\partial y}=0 \quad \text { on } \quad y=-\epsilon,
$$

and

$$
\frac{\partial \phi}{\partial t}+\frac{1}{2}|\nabla \phi|^{2}=0, \frac{\partial \phi}{\partial n}=v_{n} \quad \text { on } \quad y=h^{ \pm}(x, t),
$$

where $\partial / \partial n$ denotes the outward normal derivative to, and $v_{n}$ the normal velocity of, the relevant free surface. Note that we have implicitly assumed that there is no vortex sheet separating the water originally in the droplet from that originally in the layer; this assumption might not be justified if the region of initial contact were a segment 
rather than a point. The initial and far field conditions are

$$
\begin{aligned}
& \phi(x, y, 0)=\left\{\begin{array}{cl}
-y, & x^{2}+(y-1)^{2}<1, \\
0, & -\epsilon<y<0 ;
\end{array}\right. \\
& x^{2}+\left(h^{+}(x, 0)-1\right)^{2}=1, h^{-}(x, 0)=0 ; \\
& \phi \rightarrow 0, h^{-} \rightarrow 0 \text { as } \quad|x| \rightarrow \infty,-\epsilon<y<0 .
\end{aligned}
$$

We shall from time to time write $\phi=\operatorname{Re}\{w(z, t)\}$, where $w$ is the complex potential and $z=x+\mathrm{i} y$.

\section{Asymptotic development}

The dimensionless model problem (2.1)-(2.6) is characterized by three length scales: the initial droplet radius, 1 , the initial layer thickness, $\epsilon$, and the nominal penetration depth, $t$. The lower unperturbed droplet surface is given by

$$
y=f(x)-t+O\left(t^{2}\right) \quad \text { as } \quad t \rightarrow 0^{+} \text {for } x, y=O(1),
$$

where $f(x)=x^{2} / 2$, so the horizontal extent of the nominal penetration region is of $O\left(t^{1 / 2}\right)$ as $t \rightarrow 0^{+}$. Hence, at times sufficiently short that this length is much smaller than the layer depth, i.e. for $t^{1 / 2} \ll \epsilon$ or $t \ll \epsilon^{2}$, the effect of the base is negligible and we may apply the Wagner theory for unequal droplet impact as in Howison et al. (1991). This theory implies that, to lowest order, $d^{-}=d^{+}=O\left(t^{1 / 2}\right)$ as $t \rightarrow 0^{+}$and that the splash jet thickness as it leaves the jet root region, $H_{J}(t)$, is of $O\left(t^{3 / 2}\right)$ as $t \rightarrow 0^{+}$. Now let us assume that for all $t \ll 1$, the splash jet thickness, of $O\left(t^{3 / 2}\right)$, is much smaller than the nominal penetration depth, $t$, which is in turn much smaller than the 'contact length', i.e. the distance $2 d^{+}(t)$ between the jet roots, of $O\left(t^{1 / 2}\right)$. We can build a self-consistent theory based on this assumption by identifying four distinguished limits as follows:

(i) $t=O\left(\epsilon^{2}\right)$, when the contact length is comparable with the layer depth;

(ii) $t=O(\epsilon)$, when the nominal penetration depth is comparable with the layer depth;

(iii) $t=O\left(\epsilon^{2 / 3}\right)$, when the jet thickness is comparable with the layer depth;

(iv) $t=O(1)$, when the penetration depth is comparable with the initial droplet radius.

In this paper we describe the asymptotic solution in stages (i)-(iii), thereby identifying the mechanics of the formation of the splash jet before the droplet undergoes global deformation in stage (iv), which must inevitably be treated numerically as described in Josserand \& Zaleski (2003), Purvis \& Smith (2004, 2005), Weiss \& Yarin (1999) and references therein. We find that stage (ii) is in fact a special case of a bona fide temporal intermediate regime between stages (i) and (iii), which is valid for all times $t$ such that $\epsilon^{2} \ll t \ll \epsilon^{2 / 3}$.

\section{Splash jet initiation, stage (i): $t=O\left(\epsilon^{2}\right)$}

\subsection{Asymptotic structure}

For dimensionless times $t=\epsilon^{2} t_{1}$, with $t_{1}$ of $O(1)$, the nominal penetration depth is much less than the layer depth of $O(\epsilon)$, which is itself comparable with the contact length, which also is of $O(\epsilon)$. We therefore set $d^{+}=\epsilon d_{1}\left(t_{1}\right)$, with $d_{1}$ of $O(1)$. The lowest-order solution is thus a generalization of the unequal droplet impact theory described in Howison et al. (1991), the only modification being the presence of the 

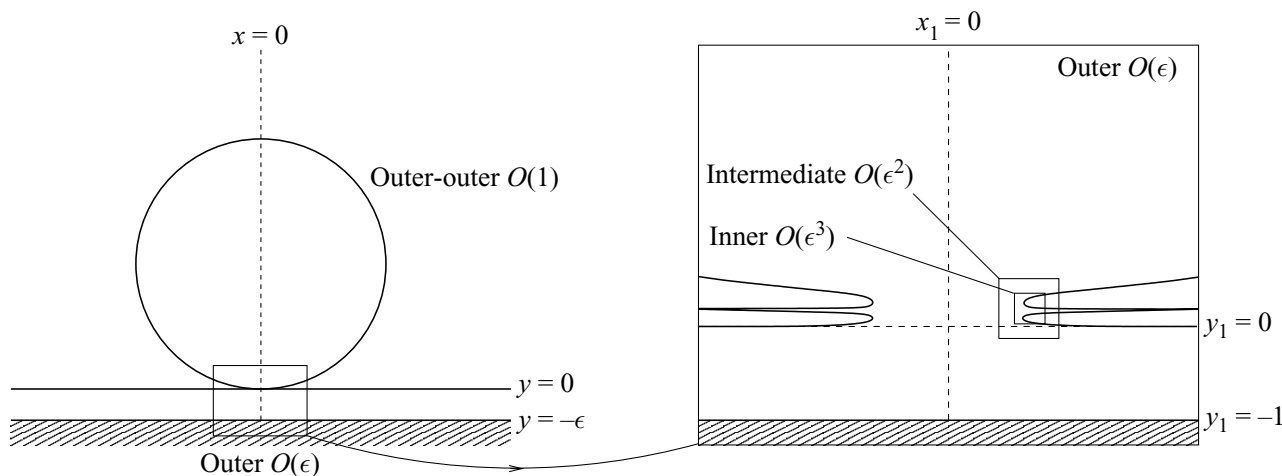

Figure 4. The asymptotic structure for $t$ of $O\left(\epsilon^{2}\right)$.

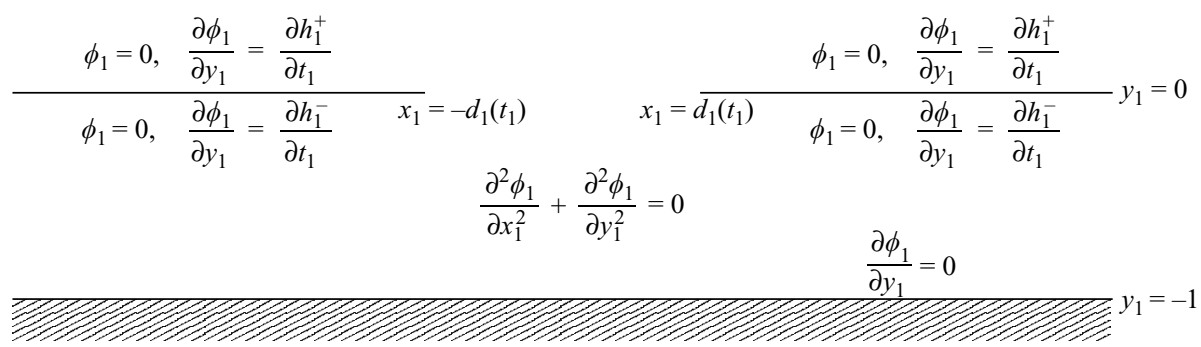

FIGURE 5. The leading-order outer problem for $t=O\left(\epsilon^{2}\right)$. In addition, the initial conditions at $t_{1}=0$ are $\phi_{1}=0$ for $-1<y_{1}<0, \phi_{1}=-y_{1}$ for $y_{1}>0, h_{1}^{-}=0, h_{1}^{+}=f\left(x_{1}\right)$ and $d_{1}=0$; the far-field conditions are $\phi_{1} \rightarrow 0$ as $|x| \rightarrow \infty$ for $-1<y_{1}<0, \phi_{1} \sim-y_{1}$ as $y_{1} \rightarrow \infty, h_{1}^{+} \rightarrow$ $f\left(x_{1}\right)-t_{1}$ and $h_{1}^{-} \rightarrow 0$ as $\left|x_{1}\right| \rightarrow \infty$; at the free points, $\phi_{1} \sim \operatorname{Re}\left\{-\mathrm{i} S_{1}\left(z_{1} \bar{\mp} d_{1}\left(t_{1}\right)\right)^{1 / 2}\right\}$ as $z_{1}=x_{1}+\mathrm{i} y_{1} \rightarrow \pm d_{1}\left(t_{1}\right)$, where $S_{1}=O(1)$ is defined in the text, and the Wagner conditions are $h_{1}^{+}\left( \pm d_{1}\left(t_{1}\right), t_{1}\right)=h_{1}^{-}\left( \pm d_{1}\left(t_{1}\right), t_{1}\right)$.

base. The asymptotic structure is depicted in figure 4. In the 'outer-outer' region, for which $z=x+\mathrm{i} y$ is of $O(1)$, there is an impulsive flow in the droplet as it reacts to an effective point force in the $y$-direction at the origin as described in Appendix A; meanwhile the splash jet mechanics are governed by the interaction between an outer flow region, in which $z$ is of $O(\epsilon)$, and inner jet root regions of size of $O\left(\epsilon^{3}\right)$ near the turnover points. These regions are joined by intermediate regions of size of $O\left(\epsilon^{2}\right)$ as shown in figure 4.

In the outer problem in figure 4 the appropriate scalings are

$$
z=\epsilon z_{1}, \phi=\epsilon \phi_{1}, h^{ \pm}=\epsilon^{2} h_{1}^{ \pm} .
$$

Expanding the potential, $\phi_{1}$, and free surfaces, $h_{1}^{ \pm}$, as asymptotic series in powers of $\epsilon$, we obtain the leading-order problem in figure 5 in which the inner jet root regions and the jets are all collapsed into the branch cuts along the $x$-axis. The far-field conditions are deduced by matching with the leading-order (uniform) flow in the outer-outer region obtained in Appendix A. The 1/2-power singularities in the potential and the Wagner conditions that the free surfaces effectively meet each other at the free points, i.e.

$$
h_{1}^{+}\left( \pm d_{1}\left(t_{1}\right), t_{1}\right)=h_{1}^{-}\left( \pm d_{1}\left(t_{1}\right), t_{1}\right)
$$


are derived by matching with the intermediate and jet root regions. These regions are sufficiently small that they only feel the effect of the base through their far-field matching conditions and, in particular, through the coefficient of the square root in the potential at the free points, denoted by $S_{1}$ in the caption to figure 5 . Hence, they are symmetric about their respective horizontal dividing streamlines and have the same structure as in solid-fluid impact at small deadrise angles, described in Howison et al. (2002). This symmetry immediately implies that, at leading order in $\epsilon$, the droplet and layer contribute equally to the flux ejected into a splash jet. We omit the technical details except to note two points. First, matching with the jet root solution as in Appendix B implies that the ejected splash jet thickness, scaled with $\epsilon^{3}$, is

$$
H_{J 1}=\frac{\pi S_{1}^{2}}{8 \dot{d}_{1}^{2}},
$$

where the overdot denotes the time derivative. Second, the far-field analysis of the jet root solution in Appendix B reveals that the coefficient of the logarithmic term in the far field is $-\dot{d}_{1} H_{J 1} / \pi$, which corresponds to the flux, $2 \dot{d}_{1} H_{J 1}$, ejected into the jet relative to the stationary frame, rather than to the the flux, $\dot{d}_{1} H_{J 1}$, ejected into the jet relative to the moving frame. We see a similar situation in the analysis of later stages of the flow below.

The evolution of the splash jet away from its root is described by the zero-gravity shallow-water equations. The theory of unequal droplet impact in Howison et al. (1991) reveals that, at leading order in $\epsilon$, the centreline of the jet is horizontal and extends to infinity; as shown in Oliver (2002), the small-time analysis in $\$ 4.2 .1$ implies that the jet thickness is of $O\left(\epsilon^{3} t_{1}^{4} /\left|x_{1}\right|^{5}\right)$ as $t_{1} \rightarrow 0^{+}$and $\left|x_{1}\right| \rightarrow \infty$.

\subsection{Analytic results for the outer problem}

Although it is possible to find the potential $\phi_{1}$ for the outer problem of figure 5, the presence of two free surfaces $y_{1}=h_{1}^{ \pm}\left(x_{1}, t_{1}\right)$, coupled by the Wagner conditions at $x_{1}= \pm d_{1}\left(t_{1}\right)$, makes the problem non-local in the sense that there is no uncoupled equation for $d_{1}\left(t_{1}\right)$. Less analytic progress is possible than for impact of a solid body of small deadrise angle on a thin fluid layer as described in Howison et al. (2004), where the corresponding codimension-two free boundary problem has only one free surface, leading to an explicit equation for the turnover point. $\dagger$

To find $d_{1}\left(t_{1}\right)$, we map the $z_{1}=x_{1}+\mathrm{i} y_{1}$ plane onto the upper-half of the $\zeta=\xi+\mathrm{i} \eta$ plane and thereby find

$$
\phi_{1}=\operatorname{Re}\left\{\frac{2 \sqrt{1-\zeta^{2}}}{\pi\left(\alpha^{2}-1\right)}\right\}, \pi z_{1}=\frac{2 \zeta}{\alpha^{2}-1}-\log \left(\frac{\zeta-1}{\zeta+1}\right) .
$$

Here, $|\xi|>1$ corresponds to the free surfaces, and $z_{1}= \pm d_{1}\left(t_{1}\right)$ corresponds to $\zeta=$ $\pm \alpha\left(t_{1}\right)$, i.e.

$$
\pi d_{1}\left(t_{1}\right)=\frac{2 \alpha\left(t_{1}\right)}{\alpha\left(t_{1}\right)^{2}-1}-\log \left(\frac{\alpha\left(t_{1}\right)-1}{\alpha\left(t_{1}\right)+1}\right) ;
$$

$d_{1}$ decreases with $\alpha$, with $d_{1} \rightarrow \infty$ as $\alpha \rightarrow 1^{+}$and $d_{1} \rightarrow 0^{+}$as $\alpha \rightarrow \infty$.

$\dagger$ Unfortunately, the "displacement potential" approach of Korobkin \& Pukhnachov (1988), which uses the time integral of the velocity potential, suffers the same disadvantages. 


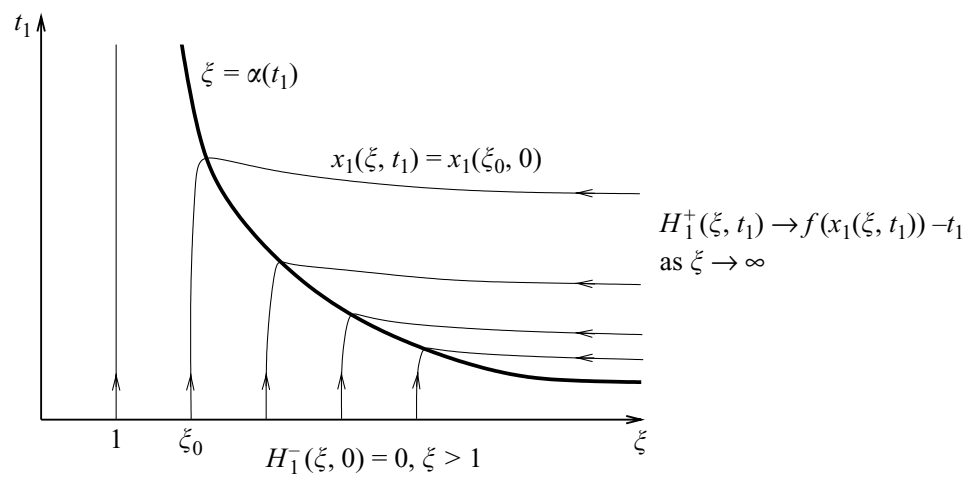

FiguRE 6. Schematic of the characteristic diagram for (4.6). The arrows indicate the direction of information flow. By (4.7), the characteristics are horizontal on the free boundary $\xi=\alpha\left(t_{1}\right)$.

Since the $x_{1}$ - and $\xi$-coordinates parameterizing the right-hand free surfaces are related by $(4.3 b)$ through

$$
\pi x_{1}(\xi ; \alpha)=\frac{2 \xi}{\alpha^{2}-1}-\log \left(\frac{\xi-1}{\xi+1}\right), \quad \xi>1,
$$

the kinematic conditions on $y_{1}=0 \pm, x_{1}>d_{1}$ in figure 5 imply the equations

$$
\frac{\partial x_{1}}{\partial \xi} \frac{\partial H_{1}^{ \pm}}{\partial t_{1}}-\frac{\partial x_{1}}{\partial t_{1}} \frac{\partial H_{1}^{ \pm}}{\partial \xi}=\frac{\partial \phi_{1}}{\partial \eta} \quad \text { on } \quad \xi>1, \eta=0,
$$

where $h_{1}^{ \pm}\left(x_{1}, t_{1}\right)=H_{1}^{ \pm}\left(\xi, t_{1}\right)$ and, by (4.3a) and (4.5),

$$
\frac{\partial x_{1}}{\partial \xi}=\frac{2}{\pi\left(\alpha^{2}-1\right)}\left(\frac{\xi^{2}-\alpha^{2}}{\xi^{2}-1}\right), \frac{\partial x_{1}}{\partial t_{1}}=\frac{4 \alpha \dot{\alpha} \xi}{\pi\left(\xi^{2}-1\right)^{2}}, \frac{\partial \phi_{1}}{\partial \eta}=-\frac{2 \xi}{\pi\left(\alpha^{2}-1\right)\left(\xi^{2}-1\right)^{1 / 2}} .
$$

The original kinematic conditions in figure 5, and therefore the quasi-linear first-order partial differential equations (4.6), have characteristics on which $x_{1}\left(\xi, t_{1}\right)=$ constant. As illustrated in figure 6 and recalling that $\alpha(0)=\infty$ as $d_{1}(0)=0$, we expect to solve (4.6) with the initial condition

$$
H_{1}^{-}(\xi, 0)=0 \text { for } \xi>1,
$$

and the far-field matching condition

$$
H_{1}^{+} \sim f\left(x\left(\xi, t_{1}\right)\right)-t_{1} \text { as } \xi \rightarrow \infty .
$$

Lastly, the Wagner condition is

$$
H_{1}^{-}=H_{1}^{+} \quad \text { on } \quad \xi=\alpha\left(t_{1}\right),
$$

where the codimension-one free boundary, $\xi=\alpha\left(t_{1}\right)$, is determined as part of the solution. This problem is 'non-local' because the characteristic equations have a nonlocal dependence on the a priori unknown location of $\xi=\alpha\left(t_{1}\right)$. A numerical solution of the potential problem in figure 5 is given in Purvis \& Smith (2005).

\subsubsection{Small- and large-time limits}

In Appendix $\mathrm{C}$ we show that, as $t_{1} \rightarrow 0^{+}$, the base has no leading-order effect in a region of size $O\left(t_{1}^{1 / 2}\right)$ near the point of impact in figure 5. In particular, we show 
that in this region the leading-order flow evolves according to the theory of unequal droplet impact described in Howison et al. (1991) and deduce that for $f\left(x_{1}\right)=x_{1}^{2} / 2$ the leading-order location of the free point is given by

$$
d_{1} \sim 2 t_{1}^{1 / 2} \text { as } t_{1} \rightarrow 0^{+}
$$

with $\alpha \sim 4 / \pi d_{1}$ as $d_{1} \rightarrow 0^{+}$. We note that at leading order the analysis in Appendix $\mathrm{C}$ also implies that

$$
h_{1}^{ \pm}\left(d_{1}\left(t_{1}\right), t_{1}\right) \sim \frac{1}{2}\left(f\left(d_{1}\left(t_{1}\right)\right)-t_{1}\right) \quad \text { as } \quad t_{1} \rightarrow 0^{+} .
$$

Hence, the vertical distance between a jet root near $x_{1}= \pm d_{1}\left(t_{1}\right)$ and the unperturbed layer surface $\left(y_{1}=0\right)$ is equal, to lowest order, to the vertical distance between the jet root and the unperturbed droplet surface $\left(y_{1}=\epsilon\left(f\left(x_{1}\right)-t_{1}\right)\right)$ as $t_{1} \rightarrow 0^{+}$. Because the jet roots do not lie on the unperturbed layer surface to lowest order, it is necessary to account for the first-order correction to the (vertical) location of the turnover points by introducing intermediate regions as in $\S 4.1$.

In Appendix D we show that, as $t_{1} \rightarrow \infty$, the fluid layer has no leading-order effect in a region of size of $O\left(t_{1}^{1 / 2}\right)$ containing both jet roots in figure 5, in the sense that the leading-order flow is the same as if the base were dry, with the leading-order location of the free point being given by

$$
d_{1} \sim 2 t_{1}^{1 / 2} \quad \text { as } \quad t_{1} \rightarrow \infty
$$

with $\alpha \sim 1+1 / \pi d_{1}$ as $d_{1} \rightarrow \infty$. Moreover, we show in Appendix D that the large-time limit of stage (i) matches with the small-time limit of stage (ii), which we describe below.

\subsection{The flux into the jet}

The coefficient of the square root in the potential in the caption to figure 5 is given by

$$
S_{1}=\left(\frac{2 \alpha}{\pi\left(\alpha^{2}-1\right)}\right)^{1 / 2} \sim\left\{\begin{array}{llll}
\left(d_{1} / 2\right)^{1 / 2} & \text { as } & d_{1} \rightarrow 0^{+} & (\alpha \rightarrow \infty), \\
\left(d_{1}\right)^{1 / 2} & \text { as } & d_{1} \rightarrow \infty & \left(\alpha \rightarrow 1^{+}\right),
\end{array}\right.
$$

in terms of which the jet thickness (scaled with $\epsilon^{3}$ ) is given by $\pi S_{1}^{2} / 8 \dot{d}_{1}^{2}$. The speed of the fluid entering the jet (scaled with $\epsilon^{-1}$ ) is $2 \dot{d}_{1}$ in the stationary frame. Hence, in this frame, the leading-order flux into the jet (scaled with $\epsilon^{2}$ ) is

$$
Q_{1}=2 \dot{d}_{1} H_{J 1}=\frac{\alpha}{2\left(\alpha^{2}-1\right) \dot{d}_{1}},
$$

with the small- and large-time behaviour being given by

$$
Q_{1} \sim\left\{\begin{array}{lll}
\pi t_{1} / 4 & \text { as } & t_{1} \rightarrow 0^{+} \\
\pi t_{1} / 2 & \text { as } \quad t_{1} \rightarrow \infty
\end{array}\right.
$$

The coefficient, $S_{1}$, and therefore the flux into a jet, $Q_{1}$, are greater than if there were no base and less than if there were no layer. As the effect of the base becomes more prominent at large times, the flux into a jet tends from below to the value it would have if the base were dry.

5. Splash jet growth, stage (ii): $\epsilon^{2} \ll t \ll \epsilon^{2 / 3}$

\subsection{Asymptotic structure}

As time increases from $t=O\left(\epsilon^{2}\right)$, the geometry of figure 4 stretches in the $x$-direction, the contact region becoming long relative to the layer depth until the effect of one 


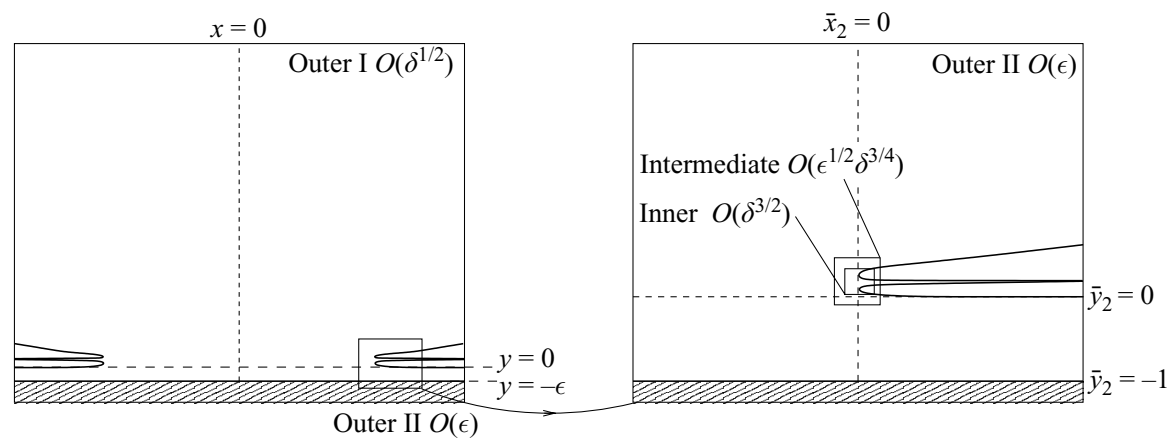

FIGURE 7. The asymptotic structure for $t$ of $O(\delta)$, where $\epsilon^{2} \ll \delta \ll \epsilon^{2 / 3}$.

$$
\phi_{2}=0, \frac{\partial \phi_{2}}{\partial y_{2}}=\frac{\partial h_{2}^{+}}{\partial t_{2}} \quad \begin{gathered}
\frac{\partial^{2} \phi_{1}}{\partial x_{2}^{2}}+\frac{\partial^{2} \phi_{2}}{\partial y_{2}^{2}}=0 \\
\frac{\partial \phi_{2}}{\partial y_{2}}=0 \\
x_{2}=-d_{2}\left(t_{2}\right)
\end{gathered} \phi_{2}=0, \frac{\partial \phi_{2}}{\partial y_{2}}=\frac{\partial h_{2}^{+}}{\partial t_{2}} \quad y_{2}=0
$$

FiguRE 8. The leading-order problem in outer region I for $t=O(\delta)$, where $\epsilon^{2} \ll \delta \ll \epsilon^{2 / 3}$. In addition, the initial conditions at $t_{2}=0$ are $\phi_{2}=-y_{1}, h_{1}^{+}=f\left(x_{2}\right)$ and $d_{2}=0$; the far-field conditions are $\phi_{2} \sim-y_{2}$ as $y_{2} \rightarrow \infty, h_{2}^{+} \rightarrow f\left(x_{2}\right)-t_{2}$ as $\left|x_{2}\right| \rightarrow \infty$; at the free points, $\phi_{2} \sim \operatorname{Re}\left\{-\mathrm{i} S_{2}\left(z_{2} \mp d_{2}\left(t_{2}\right)\right)^{1 / 2}\right\}$ as $z_{2}=x_{2}+\mathrm{i} y_{2} \rightarrow \pm d_{2}\left(t_{2}\right)$, where $S_{2}=O(1)$ is defined in the text, and the Wagner conditions are $h_{2}^{+}\left( \pm d_{2}\left(t_{2}\right), t_{2}\right)=0$.

jet root is not felt by the other. For intermediate times $t=\delta t_{2}$, where $\epsilon^{2} \ll \delta \ll \epsilon^{2 / 3}$, the asymptotic structure may therefore be deduced directly from the large-time limit of the codimension-two free boundary problem in figure 5, although, as described in Appendix D, it is in practice quicker to proceed as for the solid-fluid impact described in Howison et al. (2002).

The asymptotic structure is depicted in figure 7. The outer region (labelled with "I" in this section) has now grown in size to be of $O\left(\delta^{1 / 2}\right)$ rather than $O(\epsilon)$ although, for $x, y$ of $O(1)$, which we term the outer-outer region, the bulk flow in the droplet is again that of a response to a point force; this is equivalent to a dipole in the potential, as described in Appendix A. However, when $x \mp d^{+}(t)=O(\epsilon), y=O(\epsilon)$, the perturbed droplet boundary and the perturbed layer elevation both effectively collapse onto $y=0,|x|>d^{+}$, the base now only exerting a leading-order effect on the flow in the outer regions II which are comparable in size to the layer thickness. Two intermediate regions are again required to match systematically with the inner jet root regions, both of which have the same leading-order structure and solution as in the earlier stage (i), although they have now grown in size to be respectively of $O\left(\epsilon^{1 / 2} \delta^{3 / 4}\right)$ and of $O\left(\delta^{3 / 2}\right)$, rather than $O\left(\epsilon^{2}\right)$ and $O\left(\epsilon^{3}\right)$.

In region $I$ in figure 7 the appropriate scalings are

$$
d^{+}=\delta^{1 / 2} d_{2}, \quad z=\delta^{1 / 2} z_{2}, \phi=\delta^{1 / 2} \phi_{2}, h^{+}=\delta h_{2}^{+},
$$

with $h_{2}^{-}$being exponentially small. The resulting leading-order problem is depicted in figure 8 . Since the flow in the fluid layer is negligible to lowest order (with the layer 


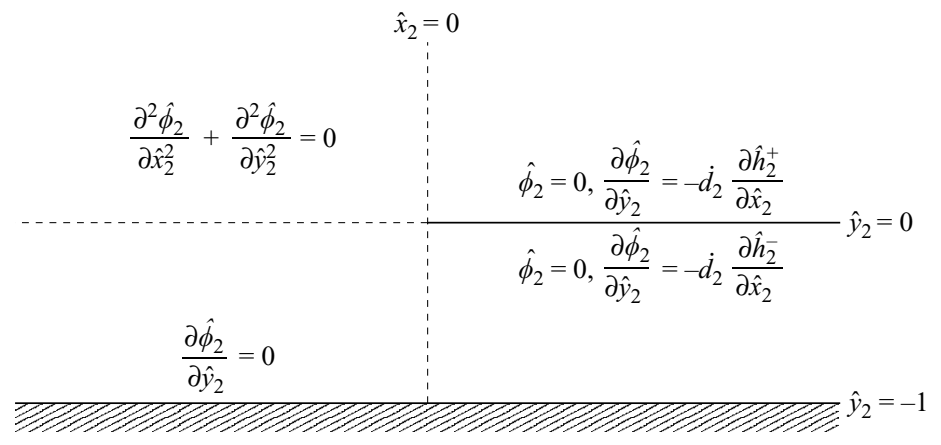

Figure 9. The leading-order problem in the right-hand outer region II for $t=O(\delta)$, where $\epsilon^{2} \ll \delta \ll \epsilon^{2 / 3}$. In addition, the far-field conditions are $\hat{\phi}_{2} \sim \operatorname{Re}\left\{-\mathrm{i} S_{2} \hat{z}_{2}{ }^{1 / 2}\right\}$ as $\hat{y}_{2} \rightarrow \infty$, where $S_{2}=O(1)$ is the same as in the caption to figure $8, \hat{w}_{2} \rightarrow 0$ as $\hat{x}_{2} \rightarrow \infty$ for $-1<\hat{y}_{2}<0, \hat{h}_{2}^{+} \rightarrow\left(2 d_{2} \hat{x}_{2}\right)^{1 / 2} / \dot{d}_{2}$ and $\hat{h}_{2}^{-} \rightarrow 0$ as $\hat{x}_{2} \rightarrow \infty$; at the origin, $\hat{\phi}_{2} \sim \operatorname{Re}\left\{-\mathrm{i} \hat{S}_{2} \hat{z}_{2}^{1 / 2}\right\}$ as $\hat{z}_{2}=\hat{x}_{2}+\mathrm{i} \hat{y}_{2} \rightarrow 0$, where $\hat{S}_{2}=O(1)$ is defined in the text, and the Wagner conditions are $\hat{h}_{2}^{+}\left(0, t_{2}\right)=\hat{h}_{2}^{-}\left(0, t_{2}\right)$.

elevation being exponentially small), this leading-order flow is almost the same as the corresponding outer problem for droplet impact on a dry base. The only difference is that here the appropriate Wagner conditions, namely

$$
h_{2}^{+}\left( \pm d_{2}\left(t_{2}\right), t_{2}\right)=0,
$$

are derived by matching with region II in figure 7, rather than by matching directly into the jet root, which is the only inner region for dry impact.

In region II in figure 7 the appropriate scalings are

$$
z_{2}=d_{2}+\epsilon \delta^{-1 / 2} \hat{z}_{2}, \quad \phi_{2}=\epsilon^{1 / 2} \delta^{-1 / 4} \hat{\phi}_{2}, h_{2}^{+}=\epsilon^{1 / 2} \delta^{-1 / 4} \hat{h}_{2}^{+}, h^{-}=\epsilon^{1 / 2} \delta^{3 / 4} \hat{h}_{2}^{-} .
$$

The resulting leading-order problem is depicted in figure 9, in which the far-field, near-field and appropriate Wagner conditions are derived as in stage (i); in particular

$$
\hat{h}_{2}^{+}\left(0, t_{2}\right)=\hat{h}_{2}^{-}\left(0, t_{2}\right)
$$

Finally, matching region II with the inner regions exactly as in stage (i), we find that the jet thickness (scaled with $\delta^{3 / 2}$ ) is

$$
H_{J 2}=\frac{\pi \hat{S}_{2}^{2}}{8 \dot{d}_{2}^{2}}
$$

where $\hat{S}_{2}$ is the coefficient of the square root in the potential in region II in figure 9 . It remains to solve the outer problems in regions I and II in figures 8 and 9, respectively, in order to determine $S_{2}$ and $\hat{S}_{2}$.

\subsection{Solution of the outer problems}

The unique solution to the potential problem in region I in figure 8 is given by

$$
\phi_{2}=\operatorname{Re}\left\{\left(d_{2}^{2}-z_{2}^{2}\right)^{1 / 2}\right\}, h_{2}^{+}=f\left(x_{2}\right)-\int_{0}^{t_{2}} \frac{\left|x_{2}\right| \mathrm{d} \tau}{\left(x_{2}^{2}-d_{2}(\tau)^{2}\right)^{1 / 2}},
$$

so that the Wagner conditions (5.2) imply $d_{2}=2 t_{2}{ }^{1 / 2}$ for $f\left(x_{2}\right)=x_{2}^{2} / 2$.

To find the unique travelling-wave solution of the potential problem in region II in figure 9 , we map the $\hat{z}_{2}=\hat{x}_{2}+\mathrm{i} \hat{y}_{2}$ plane onto the upper half of the $\zeta=\xi+\mathrm{i} \eta$ plane 
to find

$$
\hat{\phi}_{2}=\operatorname{Re}\left\{-\mathrm{i}\left(\frac{2 d_{2} \zeta}{\pi}\right)^{1 / 2}\right\} \text { where } \pi \hat{z}_{2}=-1+\zeta-\log \zeta .
$$

Integrating with respect to $\hat{x}_{2}$ the equations for $\hat{h}_{2}^{ \pm}$in figure 9 , and applying the Wagner condition (5.4), we find that the free surfaces are given by

$$
\hat{h}_{2}^{-}(x)=\frac{1}{\dot{d}_{2}}\left(\frac{2 d_{2} \xi}{\pi}\right)^{1 / 2} \quad \text { for } 0<\xi<1, \quad \hat{h}_{2}^{+}(x)=\frac{1}{\dot{d}_{2}}\left(\frac{2 d_{2} \xi}{\pi}\right)^{1 / 2} \text { for } \xi>1 \text {, }
$$

where, by (5.7), $\pi x(\xi)=-1+\xi-\log \xi$.

In summary, the fluid layer only has a leading-order effect in region II, where its presence modifies the coefficient of the square root in the potential from $S_{2}=\left(2 d_{2}\right)^{1 / 2}$ in the far field to $\hat{S}_{2}=d_{2}{ }^{1 / 2}$ near the jet roots; by (5.5), it is the latter coefficient that determines the flux ejected into a splash jet, as we now describe.

\subsection{The flux into the jet}

The ejected splash jet thickness, scaled with $\delta^{3 / 2}$, is given by (5.5) and so, substituting $d_{2}=2 t_{2}^{1 / 2}$ and $\hat{S}_{2}=d_{2}^{1 / 2}$, we find

$$
H_{J 2}=\frac{\pi t_{2}^{3 / 2}}{4}
$$

In the stationary frame the fluid entering the jet root moves with speed $2 \dot{d}_{2}$, scaled with $\delta^{-1 / 2}$, so the flux into a jet in this frame, scaled with $\delta$, is

$$
Q_{2}=2 \dot{d}_{2} H_{J 2}=\frac{\pi t_{2}}{2},
$$

with equal contributions from both the droplet and layer at leading order in $\epsilon$ by the symmetry of the jet root regions, as in stage (i) and as described in Appendix B.

We conclude that at intermediate times $t$ between $\epsilon^{2}$ and $\epsilon^{2 / 3}$, the flux ejected into a splash jet is, to lowest order, exactly equal to that for impact on a dry base. Since the inner regions grow with time, however, they must eventually be influenced directly by the geometry of the base rather than solely through their far-field matching conditions. This will occur when the ejected splash jet flux, of $O(\delta)$, becomes comparable with the flux, of $O\left(\epsilon \delta^{-1 / 2}\right)$, into region II from the layer, i.e. when $\delta$ is of $O\left(\epsilon^{2 / 3}\right)$. This corresponds to the asymptotic structure in figure 7 breaking down as region II, the intermediate and jet root regions all merge simultaneously to form a jet root region comparable in size to the layer depth, as we now describe.

\section{Splash jet and layer interaction, stage (iii): $t=O\left(\epsilon^{2 / 3}\right)$}

\subsection{Asymptotic structure}

When $t=\epsilon^{2 / 3} t_{3}$, with $t_{3}$ of $O(1)$, the splash mechanism undergoes a fundamental change, as the jet root region grows to be comparable in size to the layer depth. Our analysis for stages (i) and (ii) reveals that stage (iii) is the earliest time at which the jet root feels the asymmetry due to the base. The resulting asymptotic structure is depicted in figure 10. Although it is simpler than in stages (i) and (ii), the local solution in the jet root is more complicated and leads to some intriguing predictions 


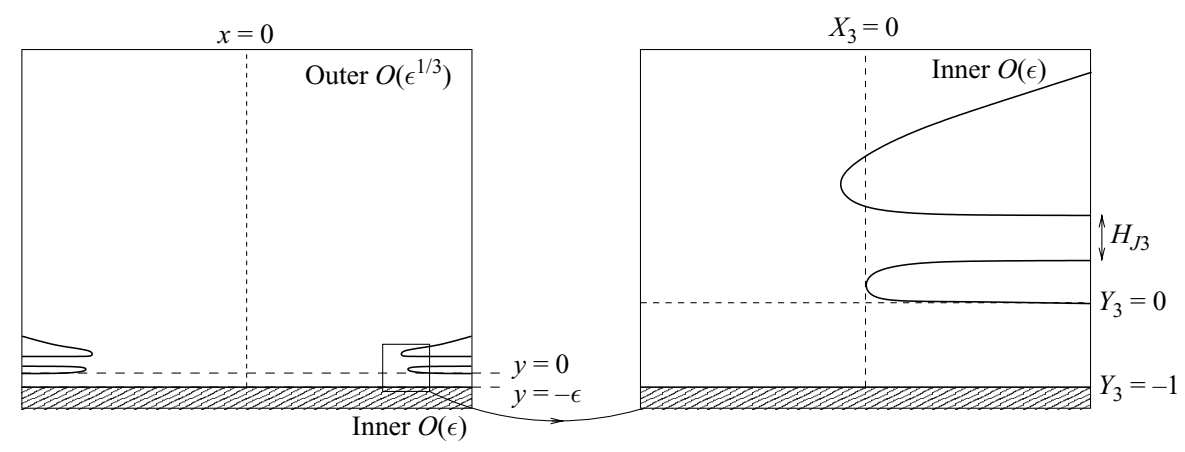

FiguRE 10. The asymptotic structure for $t$ of $O\left(\epsilon^{2 / 3}\right)$.

concerning the configuration of the relative stagnation points in the jet root region, $\dagger$ and therefore concerning the contributions of the droplet and layer to the flux ejected into a splash jet.

The appropriate scalings in the outer region in figure 10 are

$$
d^{+}=\epsilon^{1 / 3} d_{3}, \quad z=\epsilon^{1 / 3} z_{3}, \phi=\epsilon^{1 / 3} \phi_{3}, h^{+}=\epsilon^{2 / 3} h_{3}^{+},
$$

while the layer elevation, $h^{-}$, is exponentially small. The leading-order outer problem is exactly as in figure 8, although the appropriate Wagner conditions,

$$
h_{3}^{+}\left( \pm d_{3}, t_{3}\right)=0,
$$

are now derived by matching directly with the jet root region in the same way as for impact on a dry base. No intermediate regions are required to match the flows in the outer and inner regions because the jet roots lie on $y=0$ at leading order, in contrast to stages (i) and (ii).

The appropriate scalings in the inner jet root region in figure 10 are

$$
z_{3}=d_{3}+\epsilon^{2 / 3} Z_{3}, \phi_{3}=\epsilon^{1 / 3}\left(\dot{d}_{3} x_{3}+\Phi_{3}\right), h_{3}^{+}=\epsilon^{1 / 3} H_{3}^{+}, h^{-}=\epsilon H_{3}^{-},
$$

the elevations of the layer and droplet free surface now being comparable. The leading-order inner jet root problem is depicted in figure 11.

\subsection{The outer solution}

The outer solution is the same as in region I in stage (ii) in $\S 5.2$, i.e. (5.6), with the subscript 2 replaced by 3 , and $S_{3}=\left(2 d_{3}\right)^{1 / 2}$; the Wagner conditions (6.2) then give the same law of motion as in stage (ii), with $d_{3}=2 t_{3}^{1 / 2}$ for $f\left(x_{2}\right)=x_{2}^{2} / 2$.

\subsection{The inner jet root region}

The solution of the inner problem is less straightforward than before because the flow in the jet root region no longer has the symmetry of figure 16 (in Appendix B), owing to the presence of the base. Hitherto we have exploited this symmetry to solve for one half of the flow only, obtaining the other by reflection. Although there is a stagnation point in these 'deep-layer' flows, as shown in figure 16, it is on the line of symmetry and so the potential for, say, the upper half of the flow can be found by standard methods. Now, however, we must consider the location of any stagnation points relative to the base, and the two principal configurations are shown in figure 12; one has a single stagnation point within the fluid, while the other has two stagnation

$\dagger$ These are stagnation points for the flow in the moving frame, not in the stationary frame. 


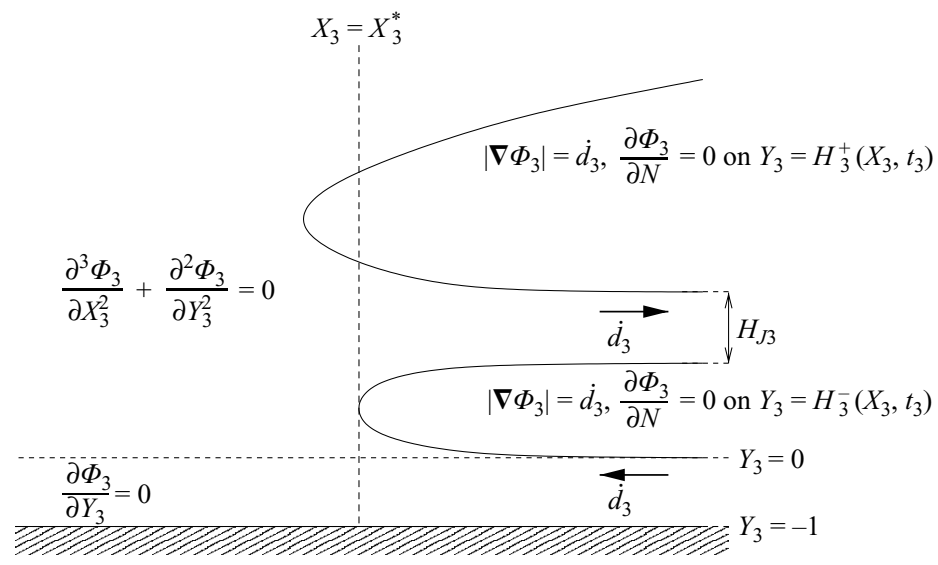

FIGURE 11. The leading-order right-hand jet root problem for $t=O\left(\epsilon^{2 / 3}\right) ; \partial / \partial N$ denotes the outward normal derivative. In addition, the far-field conditions are $W_{3} \sim-\dot{d}_{3} Z_{3}+$ $\mathrm{i} S_{3} Z_{3}^{1 / 2}+O\left(Z_{3}^{1 / 2}\right)$ as $\left|Z_{3}\right| \rightarrow \infty$, where $S_{3}=\left(2 d_{3}\right)^{1 / 2} ; H_{3}^{+} \sim S_{3} X_{3}^{1 / 2} / \dot{d}_{3}$ and $H_{3}^{-} \rightarrow 0$ as $X_{3} \rightarrow \infty$.
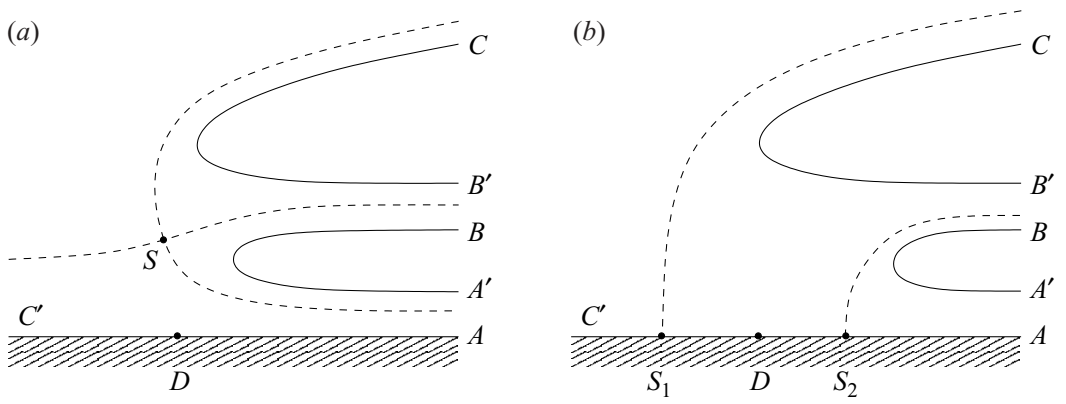

FIGURE 12. Stagnation-point configurations.

points on the wall $Y_{3}=-1$. (A third configuration, with a double stagnation point on the wall, occurs instantaneously in the transition from one stagnation point to two, and we do not discuss it in detail.) For small times $t_{3}$, matching back to the previous solutions shows that we have one stagnation point and, as we show below, there is a finite time $t_{3}^{*}$ at which this stagnation point 'touches down' on the wall $Y_{3}=-1$ and splits into two. Until this happens, some of the fluid from the initial layer remains under the impacting drop, but afterwards all the fluid from the layer is ejected in the splash jet.

\subsubsection{Flow with one stagnation point}

We write $W_{3}=\Phi_{3}+\mathrm{i} \Psi_{3}$ for the complex potential of the flow, and take $\Psi_{3}=0$ on the lower free surface $A^{\prime} B$, whence $\Psi_{3}=Q_{J 3}=\dot{d}_{3} H_{J 3}$ on the upper free surface $B^{\prime} C$; note that this is the flux into the jet in the moving frame, while the flux in the stationary frame is $Q_{3}=2 Q_{J 3}$. Also $\Psi_{3}=Q_{B 3}=\dot{d}_{3}$ on the base $C^{\prime} A$. The plane of the hodograph variable $W_{3}^{\prime}=\mathrm{d} W_{3} / \mathrm{d} Z_{3}=U_{3}-\mathrm{i} V_{3}$ is shown on figure $13(a)$; the point $D$ is where $U_{3}$ achieves its minimum value $U_{3}^{m}$, say. The hodograph plane is mapped onto 



FIgURE 13. Hodograph planes with $(a)$ one and $(b)$ two stagnation points.

the upper half- $\zeta$-plane by

$$
\zeta=\left(\gamma^{2}-\left(\frac{\dot{d}_{3}+W_{3}^{\prime}}{\dot{d}_{3}-W_{3}^{\prime}}\right)^{2}\right)^{1 / 2}
$$

where

$$
\gamma=\frac{\dot{d}_{3}-U_{3}^{m}}{\dot{d}_{3}+U_{3}^{m}}
$$

and the branch is defined such that $\zeta \rightarrow-\gamma$ as $W_{3}^{\prime} \rightarrow-\dot{d}_{3} ; \gamma$ lies between 0 and 1 , and $\gamma=1$ corresponds to touchdown. In this mapping, the point $B$ is mapped to infinity, $C$ to $-\gamma, D$ to 0 and $A$ to $\gamma$. Because $W_{3}^{\prime}=0$ at the stagnation point $S$, it is mapped to the point $\zeta_{S}=\mathrm{i}\left(1-\gamma^{2}\right)^{1 / 2}$.

As the boundary of the flow domain in the potential plane (a two-sheeted Riemann surface which is not shown here) is bounded by straight lines $\Psi_{3}=$ constant, the relation between $W_{3}$ and $\zeta$ is found via the Schwarz-Christoffel formula

$$
\frac{\mathrm{d} W_{3}}{\mathrm{~d} \zeta}=K \frac{\left(\zeta-\zeta_{S}\right)\left(\zeta-\bar{\zeta}_{S}\right)}{(\zeta+\gamma)^{2}(\zeta-\gamma)}=K \frac{\zeta^{2}+\left|\zeta_{S}\right|^{2}}{(\zeta+\gamma)^{2}(\zeta-\gamma)}
$$

where $K$ is an unknown scaling constant; the zeros in the numerator of this expression take account of the stagnation point and the denominator gives the correct behaviour at $A$ and $C$. This formula also gives the solution of the boundary-value problem in figure 14(a), and consideration of the jumps in $\Psi_{3}$ at $\zeta=\gamma$ and $\zeta=\infty$ yields the two relations

$$
\frac{\pi K}{4 \gamma^{2}}=Q_{B 3}, \quad \pi K=Q_{J 3},
$$

respectively among the three unknowns $K, \gamma$ and $Q_{J 3}$ (or $H_{J 3}$ ); the jump at $\zeta=-\gamma$ is consistent with these and provides no new information.

The final relation needed comes from matching with the outer flow. The inner limit of the one-term outer solution, given in $\S 6.2$, is $W_{3} \sim-\dot{d}_{3} Z_{3}+i\left(2 d_{3}\right)^{1 / 2} Z_{3}^{1 / 2}$, so that the matching condition is

$$
W_{3}^{\prime} \sim-\dot{d}_{3}+\mathrm{i}\left(d_{3} / 2\right)^{1 / 2} Z_{3}^{-1 / 2}+O\left(Z_{3}^{-1}\right),
$$

which is sufficient to specify $W_{3}^{\prime}$ uniquely. The local behaviour of the inner solution, near the corresponding point $\zeta=-\gamma$, is found by expanding (6.4) to give

$$
W_{3}^{\prime} \sim-\dot{d}_{3}+(8 \gamma)^{1 / 2} \dot{d}_{3}(\zeta+\gamma)^{1 / 2}+O(\zeta+\gamma)
$$


(a)

- $\mathrm{S}: \zeta=\zeta_{S}=\mathrm{i}\left(1-\gamma^{2}\right)^{1 / 2}$

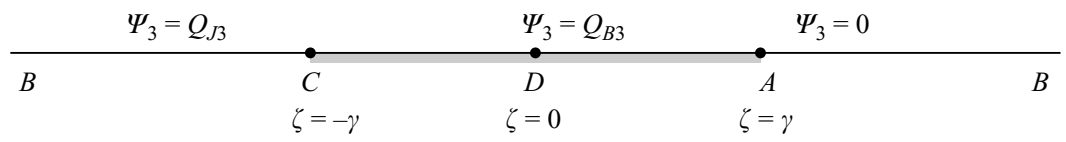

(b)

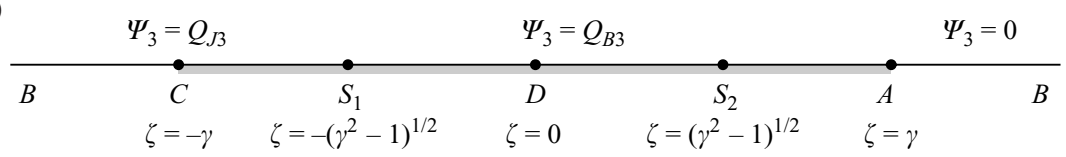

Figure 14. The $\zeta$ planes for $(a)$ one and $(b)$ two stagnation points.

then, writing $\mathrm{d} Z_{3} / \mathrm{d} \zeta=\left(\mathrm{d} W_{3} / \mathrm{d} \zeta\right) / W_{3}^{\prime}$, we use (6.5) to show that

$$
Z_{3} \sim-\frac{K}{2 \gamma \dot{d}_{3}(\zeta+\gamma)}+O\left((\zeta+\gamma)^{-1 / 2}\right)
$$

When this is used to replace $\zeta+\gamma$ in (6.6) to give the far-field behaviour of the inner solution, $\uparrow$ and the result compared with (6.6), we find the third relation $K=d_{3} / 8 \dot{d}_{3}$, from which we immediately deduce that

$$
Q_{J 3}=\frac{\pi d_{3}}{8 \dot{d}_{3}}, \quad \gamma^{2}=\frac{\pi d_{3}}{32 \dot{d}_{3}^{2}} .
$$

Remarkably, even though there is a strong interaction between the jet root and the base, the jet thickness, $Q_{J 3} / \dot{d}_{3}$, is independent of the layer thickness. The total flux into the jet is also determined by the outer solution, and has the same value as for dry impact. However, the calculation below reveals that the proportions of fluid in the jet that come from the drop and the layer, which are determined by the value of $\Psi_{3}$ on the dividing streamline, vary as time increases. The value of $\gamma$ increases from 0 at $t_{3}=0$ to 1 at touchdown, while the proportion from the layer is a decreasing function of $\gamma$, starting at $1 / 2$ when $\gamma=0$, thereby matching back to the stage (ii) solution in which the jet is composed equally of fluid from the drop and the layer. Moreover, as $\gamma \rightarrow 1$ (touchdown), this proportion tends to $1 / 4$.

In order to show this, we integrate (6.6) (with $K$ replaced by $Q_{J 3} / \pi$ ) to find

$$
W_{3}=W_{30}+\frac{Q_{J 3}}{4 \pi \gamma^{2}}\left(\log (\zeta-\gamma)+\left(4 \gamma^{2}-1\right) \log (\zeta+\gamma)+\frac{2 \gamma}{\zeta+\gamma}\right),
$$

where $W_{30}$ is a constant whose imaginary part, $\Psi_{30}$, is the flux into the jet from the layer. Setting $\Psi_{3}=Q_{3 B}$ at $\zeta=0$ we find, after rearranging, that

$$
\frac{\Psi_{30}}{Q_{J 3}}=\left(\frac{\pi}{4}-\frac{\theta}{2}\right) \sec ^{2} \theta+\theta-\frac{1}{2} \tan \theta,
$$

$\dagger$ The inner solution $W_{3}$ also has a logarithmic term in its far-field behaviour, whose strength is determined by the other parameters of the inner flow. This term matches with the corresponding term in the one-term inner expansion of the two-term outer expansion (not treated here), and the coefficients of these terms, which match automatically, confirm the mass flux arguments below. 
where $\cos \theta=\gamma$. Standard arguments show that the right-hand side increases from $1 / 4$ at $\theta=0(\gamma=1)$ to $1 / 2$ at $\theta=\frac{1}{2} \pi(\gamma=0)$ as required.

When $f(x)=x^{2} / 2$, so that $d_{3}\left(t_{3}\right)=2 t_{3}^{1 / 2}$, we have $\gamma^{2}=\pi t_{3}^{3 / 2} / 16$, and the touchdown of the stagnation point occurs at $t_{3}=(16 / \pi)^{2 / 3}$.

In summary, the flow near the jet root has one stagnation point until the finite time at which touchdown occurs. As this flow evolves, the proportion of fluid entering the jet from the layer drops from $1 / 2$ for small times to $1 / 4$ at touchdown. We now briefly consider the flow after touchdown.

\subsubsection{Flow with two stagnation points}

With two stagnation points, as shown on figure 12(b), the procedure described above is only slightly modified. The hodograph and $\zeta$-planes are now as indicated in figures $13(b)$ and $14(b)$, and now

$$
\gamma=\frac{\dot{d}_{3}+U_{3}^{M}}{\dot{d}_{3}-U_{3}^{M}}>1,
$$

where $U_{3}^{M}$ is the maximum wall speed between the two stagnation points $S_{1}$ and $S_{2}$. These are mapped onto the real points $\zeta= \pm \zeta_{S}= \pm\left(\gamma^{2}-1\right)^{1 / 2}$ and, apart from the change that now

$$
\frac{\mathrm{d} W_{3}}{\mathrm{~d} \zeta}=K \frac{\zeta^{2}-\zeta_{S}^{2}}{(\zeta+\gamma)^{2}(\zeta-\gamma)},
$$

the matching and flux calculation go through exactly as before. The flux into the jet is still the same as for dry impact, and the principal difference in the flow is that now all the fluid from the layer is expelled via the jet. Finally, for large $t_{3}$ (large $\gamma$ ), we show in Appendix E that the distance between the stagnation points increases, with $S_{2}$ tending to $A$ in figure 12 ; the upper part of the free surface tends to a conventional jet root as shown in the upper half of figure 16, while far downstream along the jet (which is now much thicker than the layer), the lower part of the free surface forms a much smaller jet root whose configuration is also as in the upper half of figure 16 but with the flow direction reversed.

\section{Discussion}

We have provided a comprehensive decomposition of the splash jet mechanisms for the two-dimensional violent impact of an inviscid droplet on a base coated with a thin inviscid layer. We are thus able to relate the jet structure for dry impact to that for impact on an infinitely deep layer. This has enabled us to show how the fraction of the thin liquid layer in the jet decreases as time increases. At leading order in the dimensionless layer thickness, $\epsilon$, we have shown that for times $t$ such that $t \ll \epsilon^{2 / 3}$, the layer and droplet contribute equally to the flux ejected into a splash jet, while for $\epsilon^{2 / 3} \ll t \ll 1$, the layer contribution is negligible. The fraction of the layer ejected into a jet decreases from one-half to zero over times of $O\left(\epsilon^{2 / 3}\right)$ as the result of an interesting bifurcation in the location of the (relative) stagnation points in the jet root.

Although the theory discussed above is two-dimensional, it can be extended with very little difficulty to the impact of an axisymmetric drop. In the early stages (i) and (ii), the jet root region is small compared with all other length scales so that, as in the impact of a solid axisymmetric body (see Howison et al. 1991), the flow in it is to leading order two-dimensional in radial planes. Hence the inner solution is 
the same as in two dimensions, with appropriate modification of the coefficient of the square root of $z$ in the far-field inner expansion of the potential. This coefficient is determined by solving the axisymmetric versions of the relevant outer problems solved above, namely that of figure 5 in stage (i) and figure 8 in stage (ii). The former is not straightforward and the latter is a well-known problem equivalent to finding the potential of a charged circular disk (see, for example, Oliver 2002; Schmieden 1953; Vorvich \& Yudovich 1957). The latter solution can also be used in stage (iii) where, although the jet root is of the same size as the layer depth, the lateral extent of the contact set is so large that the jet root flow is again two-dimensional. The final modification needed is to calculate the response of the drop (the outer-outer flow) in the axisymmetric geometry rather than in two dimensions.

It is interesting to consider over what time scales the effects of surface tension or viscosity may be important (the effects of a trapped air layer may also be significant but we do not discuss them here). Consider first the effect of a non-zero surface tension $\sigma$. The free-surface curvature is largest in the jet root, and we expect the effect of surface tension to be characterized by the size of a local Weber number $W e_{j}=\rho r_{j} u_{j}^{2} / \sigma$ based on the radius of curvature $r_{j}$ in the jet root and the local fluid velocity $u_{j}$ there. Provided that this local Weber number is large, our model should be valid. Now in the two-dimensional configuration, for small dimensional time $t$ the effective penetration is $O(U t)$, the lateral extent of the contact set is $O\left((R U t)^{1 / 2}\right)$, and the deadrise angle is therefore $\left.O(U t / R)^{1 / 2}\right)$. Bearing in mind that the size of the jet root is the square of the deadrise angle times that of the contact set, we have $r_{j}=O\left(U^{3 / 2} R^{-1 / 2} t^{3 / 2}\right)$ and $u_{j}=O\left((R U / t)^{1 / 2}\right)$, giving

$$
W e_{j}=O\left(\frac{\rho U^{5 / 2} R^{1 / 2} t^{1 / 2}}{\sigma}\right) .
$$

Hence we expect the effects of surface tension to be negligible provided that

$$
t \gg \frac{\sigma^{2}}{\rho^{2} U^{5} R} .
$$

This result also applies to axisymmetric drop impact, as the hoop stress due to surface tension is an order of magnitude smaller than that due to the curvature in radial planes.

A similar calculation for the effects of viscosity involves the local Reynolds number $R e_{j}=u_{j} r_{j} / v$, where $v$ is the kinematic viscosity of the liquid. A similar calculation to that above shows that, until the effect of the no-slip condition at the base becomes felt, viscosity has a negligible effect provided that

$$
t \gg \frac{v}{U^{2}},
$$

independently of $R$.

Concerning the temporal validity of our model, we can add that, before 2002, we would have had to conclude our work by expressing the hope that solutions satisfying our initial conditions could indeed be physically realistic. Fortunately, Thoroddsen (2002) has reported experiments using ultra-high-speed photography in which a drop impacted a deep layer of water. Although the layer was deep, the early stages of this impact may be expected to be close to our situation. Figure $1(b)$ of that paper shows the early-stage (a few microseconds) impact of a drop of water-glycerin with a viscosity of $43 \mathrm{cP}$ and surface tension of $73 \mathrm{dyn} \mathrm{cm}^{-1}$. The drop radius was $3 \mathrm{~mm}$, with an impact velocity of $620 \mathrm{~cm} \mathrm{~s}^{-1}$. A splash jet of the kind we report can clearly 
be seen. For this situation, the time scales calculated above are $0.2 \mathrm{~ns}$ for surface tension and $1.1 \mu$ s for viscosity. Hence we conclude that our model is valid in this situation for all except a very small initial time interval, with the effects of surface tension and viscosity on the flow instantaneously after contact being sufficiently small that our initial condition may be applied. That is, the small-time limit of our stage (i) is consistent with the full model incorporating the effects of viscosity and surface tension as these latter effects for a while become insignificant with increasing time from the instant of contact. Our model may therefore be useful in parameter ranges inaccessible to numerical approaches such as that of Josserand \& Zaleski (2003).

The authors are very grateful to Alexander Korobkin for stimulating discussions concerning this problem, and to the referees and editor for helpful comments. J. M. O. acknowledges the support of the EPSRC (GR/S66701/01 and GR/S93585/01(P)). R. P. and F. T. S. thank the EPSRC and QinetiQ for support through the Faraday Partnership for Industrial Mathematics, managed by the Smith Institute, and also David Allwright, Roger Gent, David Hammond, Richard Moser and Manolo Quero for their interest and helpful discussions.

\section{Appendix A. The outer-outer region in stages (i)-(iii)}

In stage $(j)\left(j=\mathrm{i}\right.$, ii, iii) the far-field expansion of the complex potential $w_{j}\left(z_{j}, t_{j}\right)$ of the leading-order outer solution is (corresponding to region I in stage (ii))

$$
w_{j} \sim \mathrm{i} z_{j}-\frac{\mathrm{i} G_{j}\left(t_{j}\right)}{z_{j}} \text { as }\left|z_{j}\right| \rightarrow \infty,
$$

where the coefficients, $G_{j}$, of the dipole are given by

$$
G_{1}=\frac{4}{\pi^{2}\left(\alpha^{2}-1\right)}+\frac{2}{\pi^{2}\left(\alpha^{2}-1\right)^{2}}, G_{2}=d_{2}^{1 / 2} / 4, G_{3}=d_{3}^{1 / 2} / 4 .
$$

For the purposes of this Appendix we take $\delta=\epsilon^{2}$ in stage (i), $\epsilon^{2} \ll \delta \ll \epsilon^{2 / 3}$ in stage (ii) and $\delta=\epsilon^{2 / 3}$ in stage (iii), so that $z_{j}=\delta^{-1 / 2} z, w_{j}=\delta^{-1 / 2} W^{o}$ in the stage $(j)$ outer-outer region. Hence, the (two-term) matching condition near the point of impact is

$$
W^{o} \sim \mathrm{i} z-\delta \frac{\mathrm{i} G_{j}\left(t_{j}\right)}{z} \text { as }|z| \rightarrow 0 .
$$

We therefore expand the complex potential as a power series: $W=W_{0}^{o}+\delta W_{1}^{o}+O\left(\delta^{2}\right)$. Introducing polar coordinates $(r, \theta)$ centred on the unperturbed droplet centre via

$$
x=r \sin \theta, \quad y=1+r \cos \theta-\delta t_{j},
$$

and denoting the perturbed droplet surface by $r=R(\theta, t)$, we also expand $R=$ $R_{0}+\delta R_{1}+O\left(\delta^{2}\right)$. By (A 3$)$, the leading-order solution is simply uniform motion of the droplet, with $W_{0}^{o}=\mathrm{i} z$ and $R_{0}=1$, while the second-order potential problem for $\Phi_{1}^{o}=\operatorname{Re}\left\{W_{1}^{o}\right\}$ is depicted in figure 15; the flow correction is driven by its singularity at the origin.

The unique solution to the potential problem in figure 15 is

$$
\Phi_{1}^{o}=\frac{G_{j}\left(t_{j}\right)}{2}\left(\frac{x^{2}+(y-1)^{2}-1}{x^{2}+y^{2}}\right)
$$




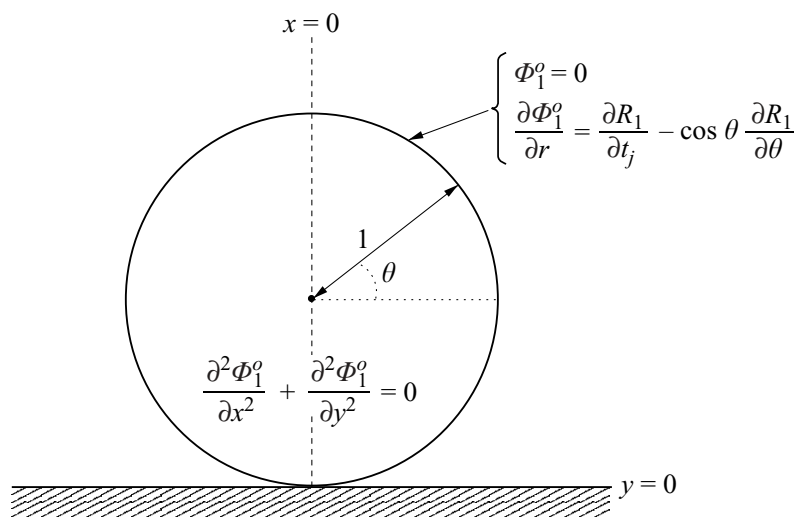

FIGURE 15. The second-order outer-outer problem in stage $(j)$. In addition, the initial conditions are $\Phi_{1}^{o}=0, R_{1}=0$ at $t_{j}=0$; at the origin $\Phi_{1}^{o} \sim-G_{j}\left(t_{j}\right) y /\left(x^{2}+y^{2}\right)$ as $x^{2}+y^{2} \rightarrow 0$.

so that on $r=1$,

$$
\frac{\partial \Phi_{1}^{o}}{\partial r}=\frac{G_{j}\left(t_{j}\right)}{2(1+\sin \theta)} .
$$

Integrating the kinematic condition for $R_{1}$ in figure 15 using the method of characteristics, we obtain the solution

$$
R_{1}\left(\theta, t_{j}\right)=\frac{1}{4} G_{j}\left(s(\theta) \mathrm{e}^{t_{j}}\right) \int_{0}^{t_{j}}\left(1-\left(1-\left(\frac{2 s(\theta) \mathrm{e}^{t_{j}-\tau}}{1+s^{2}(\theta) \mathrm{e}^{2\left(t_{j}-\tau\right)}}\right)^{2}\right)^{1 / 2}\right)^{-1} \mathrm{~d} \tau,
$$

where $s(\theta)=\tan \left(\frac{1}{2} \theta+\frac{1}{4} \pi\right)$. Finally, we note that (A 5) implies that the droplet free surface in the outer-outer and outer regions automatically match to two terms; the far-field expansion of the one-term outer expansion is given by

$$
h_{j} \sim f\left(x_{j}\right)-t_{j}-\frac{\int_{0}^{t_{j}} G_{j}(\tau) \mathrm{d} \tau}{x_{j}^{2}} \text { as }\left|x_{j}\right| \rightarrow \infty .
$$

\section{Appendix B. The jet root region in stages (i) and (ii)}

The jet roots have the same structure and solution in both stages (i) and (ii) and, for ease of notation, we describe the former. In the right-hand jet root region in figure 4, the appropriate scalings are (writing $z=x+\mathrm{i} y$ and $Z_{1}=X_{1}+\mathrm{i} Y_{1}$ )

$$
z=\epsilon d_{1}+\mathrm{i} \epsilon^{2} h_{1}^{ \pm}\left(d_{1}, t_{1}\right)+\epsilon^{3} Z_{1}, \phi=\epsilon^{2}\left(\dot{d}_{1} X_{1}+\Phi_{1}\right), h^{ \pm}=\epsilon^{2} h_{1}^{ \pm}\left(d_{1}, t_{1}\right)+\epsilon^{3} H^{ \pm},
$$

which lead at leading order to the Helmholtz cavity flow of figure 16. The farfield conditions are obtained by matching with the right-hand intermediate region in figure 4, in which the leading-order solution is simply the local travelling wave solution of the outer problem translated vertically by a distance, $\epsilon^{2} h_{1}^{ \pm}\left(d_{1}, t_{1}\right)$, so that the location of the square-root in the potential coincides with the jet root near $z=\epsilon d_{1}+\mathrm{i} \epsilon^{2} h_{1}^{ \pm}\left(d_{1}, t_{1}\right)$.

The far-field conditions imply that the flow is symmetric about a dividing streamline, say $Y_{1}=Y_{1}^{*}$; this is true as time increases through stage (ii) until, in stage (iii), the 


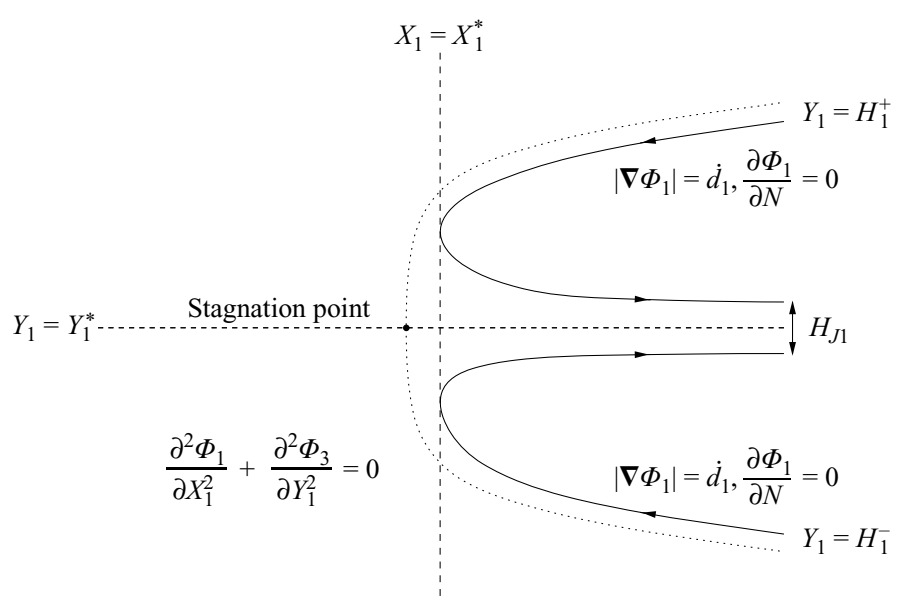

FIGURE 16. The leading-order right-hand jet root problem for $t=O\left(\epsilon^{2}\right) ; \partial / \partial N$ denotes the outward normal derivative. In addition, the far-field conditions are $W_{1} \sim-\dot{d}_{1} Z_{1}+i S_{1} Z_{1}^{1 / 2}$ as $\left|Z_{1}\right| \rightarrow \infty$ and $H_{1}^{ \pm} \sim \pm S_{1} X_{1}^{1 / 2} / \dot{d}_{1}$ as $X_{1} \rightarrow \infty$, where $S_{1}=O(1)$ is defined in the text.

jet root is large enough to be affected by the asymmetry caused by the base. The solution in $Y_{1}>Y_{1}^{*}$ and $Y_{1}<Y_{1}^{*}$ is therefore exactly the same as in the jet root region of Wagner theory for solid-fluid impact at small deadrise angles; see, for example, Howison et al. (1991). There is a single stagnation point and the parametric solution in $Y_{1}>Y_{1}^{*}$ is obtained by mapping the fluid region in $Y_{1}>Y_{1}^{*}$ to the upper half of the $\zeta$-plane. The result for the complex potential $W_{1}=\Phi_{1}+\mathrm{i} \Psi_{1}$ may be written (see, for example, E. O. Tuck 1994, personal communication)

$$
W_{1}=\frac{\dot{d}_{1} H_{J 1}}{2 \pi}(\zeta-\ln \zeta) \quad \text { where } \quad Z_{1}-Z_{1}^{*}=-\frac{H_{J 1}}{2 \pi}\left(1+\zeta+4 \zeta^{1 / 2}+\ln \zeta\right),
$$

and $Z_{1}^{*}=X_{1}^{*}+\mathrm{i} Y_{1}^{*}$ is a complex constant left unspecified by the leading-order matching, so that the solution is unique up to linear translations. In the far field (B 2) implies

$$
W_{1} \sim-\dot{d}_{1} Z_{1}-4 \mathrm{i} \dot{d}_{1}\left(\frac{H_{J 1} Z_{1}}{2 \pi}\right)^{1 / 2}-\frac{\dot{d}_{1} H_{J 1}}{\pi} \log Z_{1}+O(1) \quad \text { as } \quad\left|Z_{1}\right| \rightarrow \infty,
$$

so that the far-field matching condition for the complex potential in figure 16 implies that $4 \dot{d}_{1}\left(H_{J 1} / 2 \pi\right)^{1 / 2}=S_{1}$. Thus, the ejected jet thickness is given by (4.2) (and, similarly, by (5.5) in stage (ii)). This expression is consistent with the far-field matching condition on the free surfaces in the caption to figure 16.

\section{Appendix C. The small-time limit of the outer solution in stage (i)}

As described in $\S 3$, for $t \ll \epsilon^{2}$ the contact length is small compared with the layer depth, so in figure 5 we expect the effect of the base to be negligible in a region near the point of impact of size of $O\left(t_{1}^{1 / 2}\right)$ as $t_{1} \rightarrow 0^{+}$. Together with the Wagner theory of unequal droplet impact described in Howison et al. (1991), this suggests that the relevant (similarity) scalings as $t_{1} \rightarrow 0^{+}$are

$$
\left.\begin{array}{ll}
d_{1}=\hat{d}_{1} t_{1}^{1 / 2}+O\left(t_{1}\right), & z_{1}=t_{1}^{1 / 2} \hat{z}_{1}+O\left(t_{1}\right) \\
\phi_{1}=t_{1}^{1 / 2} \hat{\phi}_{1}\left(\hat{x}_{1}, \hat{y}_{1}\right)+O\left(t_{1}\right), & h_{1}^{ \pm}=t \hat{h}_{1}^{ \pm}\left(\hat{x}_{1}\right)+O\left(t_{1}^{3 / 2}\right),
\end{array}\right\}
$$


where $\hat{z}_{1}=\hat{x}_{1}+\mathrm{i} \hat{y}_{1}$ and the constant $\hat{d}_{1}$ is to be determined. By (4.3) and (4.4) the corresponding scalings for $\alpha$ and $\zeta$ are given by

$$
\alpha=\hat{\alpha} t_{1}^{-1 / 2}+O(1), \zeta=t_{1}^{-1 / 2} \hat{\zeta}+O(1)
$$

where, by (4.4), the constant $\hat{\alpha}=4 / \pi \hat{d}_{1}$. Moreover, expanding (4.3) as $t_{1} \rightarrow 0^{+}$we find

$$
\hat{\phi}_{1}=\operatorname{Re}\left\{\frac{2 i \hat{\zeta}}{\pi \hat{\alpha}}\right\}, \hat{z}_{1}=\frac{2}{\pi} \frac{\hat{\alpha}^{2}+\hat{\zeta}^{2}}{\hat{\alpha}^{2} \hat{\zeta}}
$$

which imply

$$
\hat{\phi}_{1}=\operatorname{Re}\left\{\frac{1}{2}\left(\mathrm{i} \hat{z}_{1}+\left(\hat{d}_{1}^{2}-\hat{z}_{1}^{2}\right)^{1 / 2}\right)\right\} ;
$$

hence, as anticipated, the leading-order flow for small $t_{1}$ is governed by the potential problem in figure 5 with the change that we replace the layer $-1<y_{1}<0$ with the half-plane $y_{1}<0$, along with the zero-flow boundary condition in the far field, $\phi_{1} \rightarrow 0$ as $y_{1} \rightarrow-\infty$.

To find $\hat{h}_{1}^{ \pm}$, we expand the kinematic boundary conditions (4.6) and substitute the first of (C3) to find for $\hat{x}_{1}>\hat{d}_{1}$

$$
\hat{h}_{1}^{ \pm}-\frac{\hat{x}_{1}}{2} \frac{\mathrm{d} \hat{h}_{1}^{ \pm}}{\mathrm{d} \hat{x}_{1}}=-\frac{1}{2}\left(1 \pm \frac{\hat{x}_{1}}{\left(\hat{x}_{1}^{2}-\hat{d}_{1}^{2}\right)^{1 / 2}}\right) ;
$$

hence, applying the far-field conditions, $\hat{h}_{1}^{+} \sim \hat{x}_{1}^{2} / 2-1$ (for $f\left(x_{1}\right)=x_{1}^{2} / 2$ ) and $\hat{h}_{1}^{-} \rightarrow 0$ as $\hat{x}_{1} \rightarrow \infty$, we find

$$
\begin{aligned}
& \hat{h}_{1}^{+}=\frac{\hat{x}_{1}^{2}}{2}-\frac{1}{2}\left(1+\frac{2}{\hat{d}_{1}^{2}}\left(\hat{x}_{1}^{2}-\hat{x}_{1}\left(\hat{x}_{1}^{2}-\hat{d}_{1}^{2}\right)^{1 / 2}\right)\right), \\
& \hat{h}_{1}^{-}=-\frac{1}{2}\left(1-\frac{2}{\hat{d}_{1}^{2}}\left(\hat{x}_{1}^{2}-\hat{x}_{1}\left(\hat{x}_{1}^{2}-\hat{d}_{1}^{2}\right)^{1 / 2}\right)\right) .
\end{aligned}
$$

To find $\hat{d}_{1}$, we simply expand the Wagner condition (4.1) to find $\hat{h}_{1}^{+}\left(\hat{d}_{1}\right)=\hat{h}_{1}^{-}\left(\hat{d}_{1}\right)$, and thus $\hat{d}_{1}=2$, i.e. (4.11) holds.

\section{Appendix D. The large-time limit of the outer solution in stage (i)}

At large times $t_{1}$ we expect the layer to have a negligible effect on the potential problem in figure 5 on length scales of $O\left(t_{1}^{1 / 2}\right)$, in the sense that the leading-order fluid response is as if the base were dry. Together with the Wagner theory for dry impact described in Howison et al. (1991), this suggests that the scalings (C 1) also apply as $t_{1} \rightarrow \infty$, with the change that $\hat{h}^{-}$is now exponentially small (corresponding to exponentially small flow in the fluid layer outside the contact set). By (4.3) and (4.4) in the large- $t_{1}$ limit, the corresponding scalings for $\alpha$ and $\zeta$ are given by

$$
\alpha=1+\hat{\alpha} t_{1}^{-1 / 2}+O\left(t_{1}^{-1}\right), \quad \zeta=t_{1}^{-1 / 2} \hat{\zeta}+O\left(t_{1}^{-1}\right),
$$

where, by (4.4), the constant $\hat{\alpha}$ is now equal to $1 / \pi \hat{d}_{1}$. Moreover, expanding (4.3) as $t_{1} \rightarrow \infty$ we find

$$
\hat{\phi}_{1}=\operatorname{Re}\left\{\hat{d}_{1}\left(1-\hat{\zeta}^{2}\right)^{1 / 2}\right\}, \quad \hat{z}_{1}=\hat{d}_{1} \hat{\zeta}
$$


and thus

$$
\hat{\phi}_{1}=\operatorname{Re}\left\{\left(\hat{d}_{1}^{2}-\hat{z}_{1}^{2}\right)^{1 / 2}\right\}
$$

hence, as anticipated, the leading-order flow is exactly as if the base were dry.

To find the leading-order perturbation to the droplet free surface and the leadingorder locations of the free points we proceed as in Appendix C. This reveals that for $f\left(x_{1}\right)=x_{1}^{2} / 2$ and $\hat{x}_{1}>\hat{d}_{1}$,

$$
\hat{h}_{1}^{+}=\frac{\hat{x}_{1}^{2}}{2}-\frac{2}{\hat{d}_{1}^{2}}\left(\hat{x}_{1}^{2}-\hat{x}_{1}\left(\hat{x}_{1}^{2}-\hat{d}_{1}^{2}\right)^{1 / 2}\right),
$$

while the Wagner condition (4.1) implies that now $\hat{h}_{1}^{+}\left(\hat{d}_{1}\right)=0$, so that again $\hat{d}_{1}=2$, i.e. (4.12) holds.

To match the flow in this large outer region of size of $O\left(t_{1}^{1 / 2}\right)$ with the exponentially small flow in the fluid layer outside the contact set (and concomitantly the free-surface profiles $\hat{h}^{ \pm}$), it is necessary to determine the flow structure near to a turnover point in a region comparable in size to the layer depth. To do so it is perhaps easier to proceed directly as described in $\S 5$, which motivates the following scalings:

$$
\left.\begin{array}{ll}
d_{1}=\hat{d}_{1} t_{1}^{1 / 2}+\frac{1}{2 \pi} \log t_{1}+\hat{d}_{11}+O(1), & z_{1}=d_{1}+\hat{Z}_{1}+O(1), \\
\phi_{1}=t_{1}^{1 / 4} \hat{\Phi}_{1}\left(\hat{X}_{1}, \hat{Y}_{1}\right)+O\left(t_{1}^{1 / 4}\right), & h_{1}^{ \pm}=t_{1}^{3 / 4} \hat{H}_{1}^{ \pm}+O\left(t_{1}^{3 / 4}\right), \\
\alpha=1+\hat{\alpha} t_{1}^{-1 / 2}+\hat{\alpha}_{1} t_{1}^{-1}+O\left(t_{1}^{-1}\right), & \zeta=1+t_{1}^{-1 / 2} \hat{\alpha} \hat{\zeta}+O\left(t_{1}^{-1 / 2}\right),
\end{array}\right\}
$$

where $\hat{Z}_{1}=\hat{X}_{1}+\mathrm{i} \hat{Y}_{1}$ and we leave the order-unity constants $\hat{d}_{11}$ and $\hat{\alpha}_{1}$ unspecified except to note that substituting (D 5) into (4.3) and expanding we find that $\hat{\Phi}_{1}$ is given by (5.7) provided $\pi \hat{d}_{11}=\hat{\alpha}_{1} / \hat{\alpha}-\hat{\alpha} / 2$. We conclude that, as alluded to above, the large-time limit of stage (i) matches with the small-time limit of stage (ii).

\section{Appendix E. The large-time limit of the jet root solution in stage (iii)}

By finding $\mathrm{d} Z_{3} / \mathrm{d} \zeta$ and integrating, we have that

$$
Z_{3}=Z_{30}+K\left(\log (\zeta+\gamma)+\frac{1}{4 \gamma^{2}} \log \left(\frac{\zeta+\gamma}{\zeta-\gamma}\right)-\frac{1-4\left(\gamma^{2}-\zeta^{2}\right)^{1 / 2}}{2 \gamma(\zeta+\gamma)}\right)
$$

where $Z_{30}$ is a constant. The stagnation points correspond to $\zeta= \pm\left(\gamma^{2}-1\right)^{1 / 2}$, and their separation is

$$
K\left(\log \left(\frac{\gamma+\left(\gamma^{2}-1\right)^{1 / 2}}{\gamma-\left(\gamma^{2}-1\right)^{1 / 2}}\right)+\frac{\left(\gamma^{2}-1\right)^{1 / 2}}{\gamma}\right)
$$

As $\gamma \rightarrow \infty$, this has asymptotic behaviour $2 K \log \gamma$. If $d_{3}=2 t_{3}^{1 / 2}$, we have $K=$ $d_{3} /\left(8 \dot{d}_{3}\right)=t_{3} / 4, \gamma=t_{3}^{3 / 4} \sqrt{\pi} / 4$, and so the separation has asymptotic behaviour $3 / 8 t_{3} \log t_{3}$. A similar calculation can be carried out for the separation of the points of vertical tangency of the upper and lower free surfaces.

\section{REFERENCES}

Gent, R. W., Dart, N. P. \& Cansdale, J. T. 2000 Aircraft icing. Phil. Trans. R. Soc. Lond. A 358, 2873-2911.

Howison, S. D., Ockendon, J. R. \& Oliver, J. M. 2002 Deep- and shallow-water slamming at small and zero deadrise angles. J. Engng Maths 42, 373-388. 
Howison, S. D., Ockendon, J. R. \& Oliver, J. M. 2004 Oblique slamming, planing and skimming. J. Engng Maths 48, 321-337.

Howison, S. D., OcKendon, J. R. \& Wilson, S. K. 1991 Incompressible water-entry problems at small deadrise angles. J. Fluid Mech. 222, 215-230.

Howison, S. D. \& Oliver, J. M. 2004 A free boundary problem arising in a model for shallow water entry at small deadrise angles. Eur. J. Appl. Math. 15, 839-851.

Josserand, C. \& Zaleski, S. 2003 Droplet impact on a thin liquid film. Phys. Fluids 15, 1650-1657.

Korobkin, A. A. 1995 Impact of two bodies one of which is covered by a thin layer of liquid. J. Fluid Mech. 300, 43-58.

Korobkin, A. A. \& Pukhnachov, V. V. 1988 Initial stage of water impact. Annu. Rev. Fluid Mech. 20, 159-185.

Oliver, J. M. 2002 Water entry and related problems. DPhil thesis, University of Oxford. http:// eprints.maths.ox.ac.uk/archive/00000132/.

Purvis, R. \& Smith, F. T. 2004 Large droplet impact on water layers. Proc. 42nd Aerospace Sci. Conference, Reno, NV, USA, Jan. 5-8, 2004, Paper 2004-0414.

Purvis, R. \& Smith, F. T. 2005 Droplet impact on water layers: post-impact analysis and computations. Phil. Trans. R. Soc. Lond. A 363, 1209-1221.

SchmiedEN, C. 1953 Der Aufschlang von rotations-k Örpen auf eine Wasseroberflä she. Z. Angew. Math. Mech. 33(4), 147-151.

Thorodssen, S. T. 2002 The ejecta sheet generated by the impact of a drop. J. Fluid Mech. 451, 373-381.

Vorvich, I. I. \& Yudovich, V. I. 1957 Circular disc impact on liquid of finite depth. Prikl. Mat. Mech. 21, 525-532.

WAGNER, H. 1932 Über stoß- und gleitvorgänge an der oberfläche von flüssigkeiten (Phenomena associated with impacts and sliding on liquid surfaces). Z. Angew. Math. Mech. 12, 193-215.

WeIss, D. A. \& YARIN, A. 1999 Single droplet impact on liquid films: neck distortion, jetting, tiny bubble entrainment and crown formation. J. Fluid Mech. 385, 229-254. 